\title{
El megaproyecto de la presa EI Zapotillo como nodo centroidal de conflicto intratable. Un análisis desde la ecología política
}

\author{
The megaproject of the El Zapotillo dam as a \\ centroidal node of intractable conflict. An analysis \\ from the political ecology
}

doi: http://dx.doi.org/10.32870/

espiral.v24i69.5283

Raúl Pacheco-Vega*

\section{Resumen}

La controversia generada por el proyecto de la presa de El Zapotillo, ubicada en el estado de Jalisco, en la zona occidente de México, ha dado lugar a una serie de movilizaciones de la sociedad civil dadas las posibles afectaciones negativas. Ciertamente, en condiciones de aridez y escasez de agua, los megaproyectos de reservas de agua antropogénicos se perciben como necesarios. En este artículo se toma una visión desde la ecología política para analizar el conflicto intratable de la presa El Zapotillo, intentando examinar las relaciones de poder $y$ las desigualdades que se acentúan en un contexto en el cual las respuestas tecnocráticas para resolver problemas de estrés hídrico provienen de una serie de instituciones jerárquicas y de una estructura sistémica que toma decisiones de gobernanza del recurso hídrico desde la cúpula y no desde la base, ignorando las opiniones de las comunidades a las cuales afectan dichas decisiones.

Palabras clave: conflictos, gobernanza del agua, megaproyectos, ecología política,

El Zapotillo.

\begin{abstract}
Controversies generated by El Zapotillo dam, in the occidental state of Jalisco, México, have led to a series of civil society negative affectations. Admittedly, conditions of drought and water scarcity make megaprojects necessary. In this article, a political ecology view is taken to analyze the intractable conflict generated by El Zapotillo, in an attempt to map power relations and inequalities that are accentuated in a context where technocratic responses to solve water stress come from hierarchical institutions and systemic structure that makes governance decisions top-down and not bottom-up, ignoring communities' opinions.
\end{abstract}

Keywords: conflicts, water governance, megaprojects, political ecology, El Zapotillo.

\footnotetext{
•Profesor-Investigador del Centro de Investigación y Docencia Económica (CIDE), México. raul.pacheco-vega@cide.edu

Fecha de recepción: 09 de marzo de 2016. Fecha de aceptación: 22 de febrero de 2017.
} 


\section{Introducción}

La construcción de la presa de El Zapotillo, ubicada en el estado de Jalisco, en la zona occidente de México, ha dado lugar a una serie de movilizaciones de la sociedad civil por posibles afectaciones negativas para los habitantes de la zona. Si bien en condiciones de aridez y escasez de agua los megaproyectos de reservas de agua antropogénicos se perciben como necesarios, es importante realizar un análisis profundo y holístico sobre los impactos negativos de los mismos.

Figura 1. Ubicación de la presa de El Zapotillo

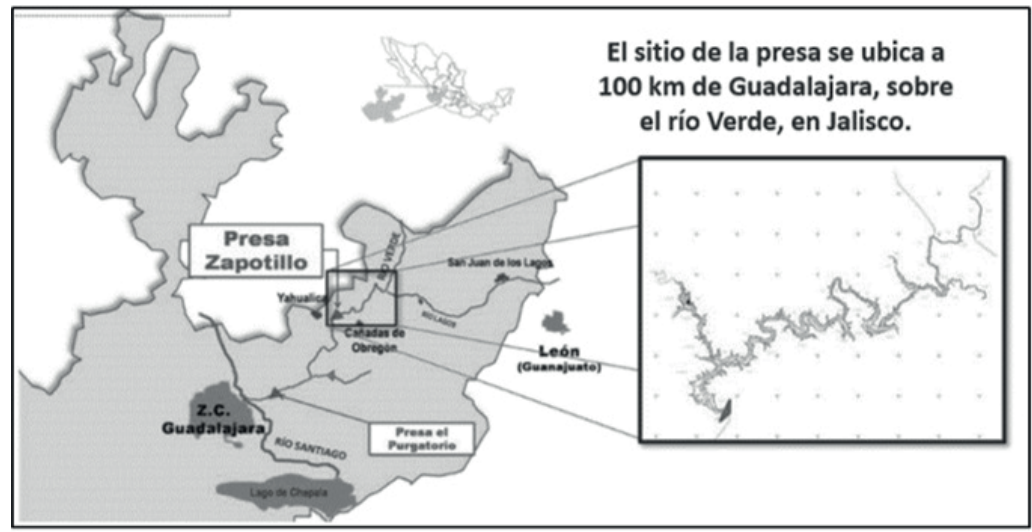

Fuente: Alonso (2015).

La creciente expansión de las ciudades, la presión del cambio de uso de suelo y las reglas de zonificación que privilegian la construcción de vivienda habitacional por encima de la continuación de la producción agrícola, la disminución de oportunidades laborales en el campo y el rápido movimiento migratorio intranacional, que transforma las comunidades anteriormente mencionadas, son situaciones que generan mayor demanda de suministro de agua en 
zonas urbanas, lo que a su vez incrementa la necesidad de encontrar alternativas de suministro que generalmente terminan siendo respuestas tecnocráticas por parte de las agencias gubernamentales encargadas del manejo del agua.

Entre dichas alternativas de política pública se encuentran los trasvases. Se conoce como "trasvase" al proyecto mediante el cual se pretende transportar agua del estado de Jalisco al estado de Guanajuato, en específico a la ciudad de León. Este proyecto surge a partir de un problema en la sobreexplotación del lago de Chapala, que es donde se obtiene parte del agua que se suministra a esta ciudad.

No se trata de un problema nuevo: ya desde el periodo presidencial de Salinas de Gortari (1988-1994) se podían observar intenciones de reconfigurar la manera en que la ciudad de León obtiene el agua (Gómez Fuentes, 2015); sin embargo, al analizar diferentes notas de periódico se puede observar que esto también ha generado un descontento por parte de diferentes actores, ya que han surgido algunos cuestionamientos sobre por qué son los habitantes de una región diferente los que se tienen que contribuir al suministro del agua, así como sobre la cuestión de quiénes son los beneficiarios de este futuro suministro, es decir, si únicamente serán beneficiados los habitantes de León o si este proyecto está pensando en las necesidades de la industria de León.

Estos movimientos intra- o intercuencas requieren de una sofisticada infraestructura ingenieril para poder transportar miles de litros de agua de una región geográfica a otra. Para poder suministrar agua continuamente, es necesario tener almacenamientos de larga duración, y por ende se hacen necesarios los megaproyectos de presas, represas y redireccionamientos de ríos y lagos. Sin embargo, a pesar de que estas macroobras generan beneficios para ciertas comunidades, también tienen asociados una gran cantidad de efectos negativos tanto para poblaciones afectadas por 
el desplazamiento originado por el proyecto ingenieril como para las mismas agencias gubernamentales, que tienen que financiar estas grandes obras frecuentemente mediante endeudamiento o, peor aún, mediante la concesión a intereses particulares de consorcios constructores.

En este artículo se conjuntan tres corrientes teóricas distintas de reciente utilización en el estudio de conflictos por el agua. En primer lugar, se utiliza el modelo IER (intratabilidad-enmarcamiento-reenmarcamiento) para explicar por qué se considera que el conflicto de El Zapotillo es intratable y qué características cumple que lo hacen intratable. En segundo lugar, se hace uso de la ecología política como marco explicativo que proporciona algunas de las razones por las cuales se generan los conflictos intratables. En particular, se utiliza la definición de "ecología política" que la establece como la economía política del cambio socioambiental (Bakker, 2003). En tercer lugar, se utiliza la metáfora conceptual de nodo centroidal con base en una aplicación visual de la teoría de redes de política pública (policy networks). El argumento es que en el estudio de los conflictos estos se convierten en el centroide de una red de política pública en donde hay grandes disparidades en el poder que tienen los diferentes nodos.

En el segundo apartado del artículo, se explica brevemente el marco de estudio de conflictos intratables propuesto de manera tácita por Elliott, Gray y Lewicki (2003), y adaptado para México por el autor del presente artículo. Este marco (IER, intratabilidad-enmarcamiento-reenmarcamiento) (Pacheco-Vega, 2014) permite evaluar la posición del conflicto en el espectro de intratabilidad, así como la determinación de las diferentes posiciones de los actores en conflicto y las posibles trayectorias de reenmarcamiento del conflicto que podrían resolver satisfactoriamente el mismo (Elliott, Gray y Lewicki, 2003). 
En el tercer apartado, se describen las contribuciones de la ecología política al estudio de conflictos urbanos por el agua y su aplicación a problemas de gobernanza de los recursos hídricos, y en el cuarto apartado se resume brevemente la aportación de la teoría de las redes de políticas públicas, explicando cómo el pensar en el conflicto intratable como el nodo centroidal puede ser una metáfora útil para mapear las diferentes fuerzas que se encuentran en conflicto para poder comenzar a estructurar un conjunto de posibles propuestas de política pública que puedan ayudar a resolver dicho conflicto. Dichas propuestas emergen de la aplicación del marco de IER al caso de estudio (Pacheco-Vega, 2014), un ejercicio que se resumen brevemente en el quinto apartado.

\section{Estudiando conflictos intratables: el modelo IER}

La realidad de los conflictos por el agua en México es multifacética y multidimensional. Las disputas por el agua van mucho más allá de las luchas por la problemática del acceso al vital liquido, extendiéndose a problemas de contaminación y afectación de la calidad de vida de comunidades marginadas, así como a las dimensiones sociales y territoriales de los mismos conflictos (Paz Salinas, 2012).

En la actualidad, todavía permanecen muchas de las confrontaciones que han sido documentadas por varios investigadores (Cairé Martínez, 2005; Becerra Pérez, Sáinz Santamaría y Muñoz Piña, 2006; Oswald Spring, 2006; Vargas-Velázquez, Mollard y Guitrón de los Reyes, 2012). No todos los conflictos por el agua son de naturaleza intratable, pero dado que una de las dimensiones de intratabilidad es precisamente la duración, los casos de estudios analizados por los autores en los volúmenes editados por Vargas-Velazquez, Mollard y Guitrón de los Reyes, así como en el trabajo de Becerra y colaboradores y de Caire, pudieran presentar más de una característica de intratabilidad. 
El modelo IER fue propuesto por Pacheco-Vega en su estudio de los conflictos intratables, basado en el marco conceptual presentado en el libro Making Sense of Intractable Environmental Conflicts. Concepts and Cases. La primera propuesta de aplicación del marco IER fue precisamente al caso de estudio de la presa de El Zapotillo (Pacheco-Vega, 2014). En los trabajos mencionados, el mapeo histórico del conflicto y el cálculo de las diferencias en percepciones positivas y negativas del megaproyecto permite comprender que los marcos posicionales individuales de los actores son extraordinariamente divergentes, en particular los de las comunidades afectadas y de las agencias gubernamentales encargadas de la gestión del agua.

La aplicación del marco IER involucra primeramente considerar el espectro de intratabilidad propuesto por Lewicky y Gray, así como por Bar-Tal, para el estudio de los conflictos que tienen larga duración, que son difíciles de resolver y que se observan como disputas en las cuales no se encuentra una posible solución negociada a corto o mediano plazo (Bar-Tal, 2000; Lewicki, Gray y Elliott, 2003; Putnam y Wondolleck, 2003). De acuerdo con Burgess y Burgess (2003), "los conflictos intratables son aquellos que se encuentran en la frontera del campo: los conflictos que testarudamente eluden resolución, aun cuando se apliquen las mejores técnicas". Para Bar-Tal, los conflictos intratables se caracterizan por ser prolongados, irreconciliables, en ocasiones violentos, de naturaleza suma-cero (es decir, hay quienes ganan y quienes pierden en el conflicto, no hay situaciones de tipo ganar-ganar), centralizados, y en ellos al menos una de las partes tiene un interés en continuarlos. Son también demandantes, estresantes, en ocasiones dolorosos, agotadores y costosos en términos materiales y humanos (Bar-Tal, 2000, p. 353).

Posteriormente, deben considerarse los diferentes marcos de referencia de cada uno de los actores participantes en el 
conflicto. El enmarcamiento debe considerarse una actividad cognitiva por parte de los agentes que son parte en el conflicto (Bar-Tal, 2000). Durante el proceso de reconocimiento de la existencia de un conflicto, los agentes participantes tratan en primer lugar de explicar la emergencia del conflicto, para posteriormente comprehender su posición en el mismo y calcular la estrategia de resolución (o de salida del conflicto, dependiendo de los objetivos de quien participa en el proceso de negociación).

Gray define el "enmarcamiento" como la actividad y el proceso por el cual se crean y representan marcos (Gray, 2003). En esta definición, el marco es la etiqueta, categoría o "cajón" mediante el cual se organizan los conceptos y nociones con respecto de un actor/agente/objeto. Por ejemplo, si un profesor otorga una calificación de 10, se le puede enmarcar como un "héroe" (o heroína). Sin embargo, si reprueba al estudiante habrá quien le enmarque como "un villano". Es decir, los marcos son volátiles, transformables, evolucionarios y cambiantes. El que un individuo enmarque un objeto de alguna forma no quiere decir que ese marco sea permanente. ${ }^{1}$

La literatura sobre reenmarcamiento (o "transformación del marco de referencia") no es precisamente nueva (Elliott, et al., 2003; Emery, Perks y Bracken, 2013), pero sí representa un cambio en la forma en la cual se piensa acerca del manejo de conflictos intratables. En lugar de pretender analizar los conflictos como juegos de suma cero (zero sum games), los estudios sobre reenmarcamiento se enfocan en alinear los marcos de las partes en conflicto o transformarlos mediante la provisión de nueva información.

Desde una perspectiva sociológica, el reenmarcamiento puede tener un carácter estratégico. Los marcos de refe-

I. Como puede observarse en esta discusión, el concepto de "marco" se refiere generalmente a la perspectiva cognitiva. 
rencia de diferentes individuos y afectados por el problema pueden ser substancialmente disímbolos. Al estar en conflicto unos marcos con otros, generan situaciones de tensión y, posiblemente, disputas. Dado que los actores en contienda pueden narrar historias diferentes con respecto a un conflicto y tener visiones contrapuestas del mismo, alinear los marcos de referencia resulta positivo no sólo para la resolución de la disputa: desde la perspectiva de un mediador (o de un tomador de decisiones en la política hidráulica) que busca la resolución de conflictos ambientales por el recurso hídrico, es importante comprender la perspectiva de cada uno de los actores involucrados en el conflicto. También es importante entender que bajo la perspectiva de reenmarcamiento puede o no haber simetría en el marco de referencia entre actores en disputa.

Finalmente, y aunque no se hace una variable explicita en el marco IER, los ámbitos de resolución (foros, avenidas) son relevantes porque pueden influir en cómo se resuelve el conflicto (Pralle, 2003; Heikkila y Schlager, 2012; Mamudu, Cairney y Studlar, 2015). Diferentes actores seleccionan diversas avenidas para resolver su conflicto. Habrá quienes consideren que pueden resolver sus diferencias en un entorno que no represente una confrontación directa (tal como una sala de mediación), o quienes consideren posible resolverlo en sus propias casas o en un foro de discusión.

\section{Aplicando ecología política al estudio de los conflictos por el agua}

La postura de ecología política asumida en este trabajo es menos radical que una neomarxista, dado que no se asume que las políticas neoliberales han sido las detonadoras de un deterioro ambiental continuo.

Por el contrario, interesa más explicar la relación causal que puede existir entre la implementación de políticas 
hidráulicas tecnocráticas y la captura de los ciudadanos en medio de los álgidos conflictos entre diferentes niveles de Gobierno y los intereses privatizadores de los consorcios proveedores tanto de infraestructura hidráulica como de servicios municipales de suministro de agua. Es importante hacer notar que las versiones de ecología política son diversas, ya que este campo se ha nutrido de las aportaciones de diferentes corrientes, como el marxismo o el postestructuralismo.

Como se ha mencionado con anterioridad, en este trabajo se aplica específicamente la ecología política urbana, ya que, como afirma Domene Gómez (2006), con la aplicación de la ecología política urbana se puede conceptualizar la urbanización como el proceso que da origen a muchos de los problemas ambientales y, al mismo tiempo, permite entender los contextos como los espacios donde los problemas socioambientales se experimentan de manera más profunda.

Este concepto ofrece un poder explicativo mayor que otros paradigmas conceptuales, debido a que involucra una multiplicidad de elementos analíticos más allá de una simple descripción del contexto urbano, del ecosistema tanto en la ciudad como periurbano, y de la economía política del desarrollo de la región. En el contexto de este artículo, la ecología política urbana permite entrelazar las realidades de disparidad de poder entre los diferentes actores que están involucrados tanto en la construcción del acueducto (incluido en el megaproyecto) como de la presa El Zapotillo. No tienen el mismo poder los activistas que defienden a las comunidades cuyos territorios serán inundados que los representantes de las agencias gubernamentales, como Conagua.

Asimismo, la ecología política urbana permite establecer una liga directa entre la escala micro-, en la cual se desarrollan las actividades extractivas (en la zona de 
Temacapulín-Acasico-Palmarejo), y la escala macro-, en la que se establecen los requerimientos de reasignación de los caudales debido a la expansión urbana tanto de la Zona Metropolitana de Guadalajara como de la de León. Por lo mismo, en ambos casos hay un reescalamiento de la problemática hídrica, lo cual crea una confrontación entre diversos intereses y una inherente transgresión de escalas. Dicha transgresión, como lo explica la ecología política urbana, pone bajo relieve las disparidades de poder entre los distintos actores involucrados en el conflicto del acueducto y la presa de El Zapotillo.

Las necesidades urbanas se privilegian por encima de los requerimientos de los agricultores, y por lo mismo se hace de primordial importancia para el Gobierno que el megaproyecto hidráulico tenga éxito. De esta manera, esta perspectiva resulta adecuada para entender la situación de la presa El Zapotillo, pues si bien las comunidades afectadas no se encuentran en un entorno urbano, las políticas que dieron origen a este megaproyecto son resultado de la urbanización mal desarrollada en el estado de Jalisco.

A pesar de la versatilidad del concepto de ecología politica, su aplicación al estudio del agua y su gestión por parte de las ciencias sociales han privilegiado primordialmente el estudio de la economía política del suministro de agua privado (Loftus y McDonald, 2001; Bakker, 2003). En este artículo, se rescatan las aportaciones de la ecología política al estudio de las relaciones de poder entre los diferentes actores involucrados en un conflicto. En específico, interesa explorar su contribución al estudio de conflictos intratables, dadas las características que hacen especiales a este tipo de disputas. El argumento presentado postula que la ecología política puede ser el recorte analítico correcto para desenmarañar la compleja madeja de relaciones de poder que están dando características de intratabilidad al conflicto bajo estudio. 
El interés primordial en el uso de ecología política reside en la comprensión de la relación entre el cambio ambiental y la evolución de los procesos sociopolíticos. También es importante hacer notar que la ecología política y -en especial- la ecología política urbana nos permiten demostrar el reescalamiento del impacto negativo de los megaproyectos (Swyngedouw, Kaïka y Castro, 2002; Swyngedouw y Heynen, 2003; Biro, 2007; Mahon y Keil, 2009).

Como indican Swyngedouw y sus colaboradores,

[...] el análisis político-ecológico del proceso de urbanización revela la naturaleza inherentemente contradictoria del proceso de cambio socio-ambiental y extrae los conflictos inevitables (o el desplazamiento de los mismos) que imbuyen al cambio socio-ambiental (Swyngedouw, et al., 2002, p. 9l I).

El análisis político-ecológico ${ }^{2}$ también evidencia la dimensión multiescalar de los problemas de cambio socioambiental. Esto ocurre en dos momentos.

Primero, los cambios sociales y ambientales urbanos están codeterminados, es decir, uno impacta al otro de manera recíproca. Como indican Swyngedouw y Heynen (2003), los procesos transformativos físicos y sociales recrean contextos con diferentes características espaciales y temporales, y viceversa, como en un proceso metabólico (Gandy, 2006). Esta dinámica (que la ecología política denomina "metabolismo", tomando una metáfora prestada de la biología) crea una situación de flujo cíclico.

Es importante hacer notar que el proceso metabólico tiene una dinámica que se encuentra incrustada en configuraciones escalares extraterritoriales o con modalidad de red. En otras palabras, existe un reescalamiento continuo

2. Se traduce aquí "political-ecological analysis" como análisis político-ecológico, mientras que se mantiene la nomenclatura de la subdisciplina como "ecología política" (political ecology). 
de las relaciones entre los diferentes actores sociales y su contexto ambiental (Swyngedouw y Heynen, 2003; Furlong, 2012; Cohen y McCarthy, 2014).

Segundo, el análisis político-ecológico enfatiza la disparidad de poder entre los actores en el contexto de una relación interescalar. Esto es particularmente relevante en el caso de megaproyectos, ya que la escala de impacto de la obra de infraestructura rebasa lo local (Monstadt, 2009) y se convierte en un problema de inequidad a múltiples escalas, no solamente gubernamentales o de política pública, sino también en el aspecto geográfico.

En el análisis presentado en páginas subsecuentes, se utilizan fundamentalmente conceptos de ecología política urbana (Swyngedouw, et al., 2002; Swyngedouw y Heynen, 2003; Monstadt, 2009). Esto es importante resaltarlo dado que el caso de estudio presenta un conflicto en el locus geográfico de la intersección de dos estados (Guanajuato y Jalisco), pero se trata de una lucha primordialmente por el suministro de agua de una región en un estado (Jalisco) que debería de suministrar el vital líquido a una zona metropolitana (Guadalajara), mientras que el agua está siendo desviada a otra zona metropolitana (León). Por lo mismo, el lente analítico geográfico forzosamente obliga a reescalar el análisis, ya que la escala del problema pasa de ser metropolitana a regional y finalmente nacional, dada la intervención de política pública de la agencia federal que maneja el agua, la Comisión Nacional del Agua (Conagua).

El argumento aquí presentado es que un lente analítico de ecología política urbana permite poner de relieve la transformación y el reescalamiento de las relaciones de poder alrededor del megaproyecto que se generan por el conflicto inherente a la construcción de la presa. La perspectiva del reescalamiento permite mostrar la continua y simbiótica evolución de las relaciones entre los diferentes actores presentes en múltiples escalas y la desigualdad de 
poder que los mismos tienen en el contexto del gobierno del agua (Pacheco-Vega, 2014).

La utilización de un lente analítico de tipo políticoecológico también enfatiza las relaciones que existen entre el ciclo hidrológico y los procesos sociales. Esta visión ha dado en llamarse el "ciclo hidrosocial" (Aguilera-Klink, Pérez-Moriana y Sánchez-García, 2000; Debbané, 2013; Linton, 2014). La conceptualización del ciclo hidrosocial permite integrar la actividad antropogénica, que genera cambios socioambientales, y los ecosistemas vistos desde una perspectiva interescalar; es decir, una visión en la cual se acepta que el acceso a recursos hídricos es dependiente de la escala geográfica, de la huella hídrica de las diferentes urbes en la región bajo análisis, y de las desigualdades, desequilibrios y asimetrías en las relaciones de poder que existen entre los varios actores involucrados en la gestión del recurso hídrico.

\section{Redes de políticas públicas: un modelo visual para comprender las relaciones entre actores}

Los estudiosos de los modelos de redes tanto en México como en el mundo tienden a utilizar el concepto para mapear las relaciones entre los diferentes actores que conforman el sistema político y de políticas públicas (Marsh, 1998). Si bien las aplicaciones son muchas, en ocasiones la definición del concepto es vaga todavía.

Particularmente problemático es que las redes de políticas pueden estar configuradas de muchas formas. En primer lugar, se puede tener una red de política pública en forma similar a las redes de coaliciones promotoras (Weible y Sabatier, 2005), al estilo más puro de Paul Sabatier. En el presente artículo se utiliza el modelo de redes de políticas públicas (policy networks) como una heurística visual y conceptual más que como el método analítico para examinar 
el problema, de forma similar a como opera el modelo de la ecología de juegos modificado por Lubell y propuesto por Norton Long en 1958 (Lubell, 2013). ${ }^{3}$

La idea del modelo de la ecología de juegos es que la gobernanza de los recursos involucra una multiplicidad de actores, instituciones y problemas. En el marco analítico que presentan Lubell y colaboradores, ellos describen seis conceptos interrelacionados: juegos de política, asuntos de política, instituciones de política, sistemas de política, actores de política y el tiempo. De hecho, la propuesta de Lubell es de los pocos recortes analíticos que involucran el horizonte temporal, o el tiempo como variable más allá que de contexto, una variable de análisis (Lubell, 2015).

$\mathrm{Al}$ centro de la red se coloca el conflicto, y alrededor del mismo se visualizan a sus diferentes actores. Un estudio más detallado de la red de política pública que se podría realizar aplicando análisis de redes sociales (social network analysis) escapa el ámbito de este documento. Sin embargo, puede ser interesante aplicar este tipo de métodos al estudio de conflictos intratables.

Los métodos que examinan redes de una manera sistemática prometen bastante. Por ejemplo, recientemente Elgin ha utilizado el análisis de redes semánticas para examinar qué actores se encuentran en contra o a favor de políticas de cambio climático (Elgin, 2015). También está el trabajo de Park y Rethemeyer, quienes calculan, mediante análisis de redes sociales, la estructura social de las redes de políticas públicas (Park y Rethemeyer, 2014). En el rubro especifico de teorías de la colaboración, se encuentran aplicaciones del análisis de redes para explicar el acceso a burócratas por parte de grupos de interés en Holanda (Beyers

3. Recientemente, Lubell ha respondido a quienes critican el modelo de ecología de juegos, ya que ciertamente es posible ver dicho marco analítico como una extensión o reformulación del concepto de policentricidad. Véase: http://environmentalpolicy.ucdavis.edu/node/379

\section{6}


y Braun, 2014) y la construcción de redes colaborativas para la gestión de parques y áreas naturales en el sur de Nevada (Schafer, 2012). En particular, el trabajo de Berardo es de relevancia para la literatura sobre colaboración en estructuras reticulares para el manejo de agua dado que su análisis demuestra que la colaboración entre organizaciones fortalece a las mismas al interior de la red y a sí mismas (Berardo, 2009). Como Berardo aclara, el reto para quienes estudian redes de política pública es comprender de manera empírica cómo es que las redes afectan las capacidades de los actores organizacionales para lograr sus objetivos.

Sin embargo, como los ejemplos mencionados demuestran, mucho del enfoque del análisis de redes está en la comprensión de modelos colaborativos, y no en el mapeo de posibles factores que incrementan el grado de intratabilidad de un conflicto. En este artículo se argumenta que cuando los actores se confrontan durante un periodo de tiempo prolongado la red de políticas públicas presenta un grado de tensión alto que no permite a los mismos alinear sus marcos de referencia y, por lo mismo, se hace virtualmente imposible la colaboración y la reducción del grado de tensión en el conflicto.

El problema fundamental que presentan los conflictos intratables es que los actores tienen poderes disímbolos y posiciones divergentes. La presuposición -incorrecta- de que la sociedad civil y los activistas tienen el mismo poder en la red de política pública que otros actores mucho más fuertes, como los grandes consorcios internacionales y nacionales de infraestructura y servicio, genera una serie de problemáticas para poder resolver el conflicto intratable. $\mathrm{Al}$ visualizar al conflicto intratable como el nodo centroidal de la red de política pública, es posible también examinar con mayor poder analítico el problema. Al colocar el conflicto en el centro de la red de política pública, se expande la visibilidad de las diferentes fuerzas que inciden en el mismo. 
Ciertamente, pudiera haber un problema conceptual al reificar un conflicto y convertirlo en un nodo de la red de política pública. Para resolver esto, en el artículo únicamente se utiliza la visualización como metáfora conceptual para iluminar las diferentes fuerzas en conflicto que colisionan en el caso de estudio. Sin embargo, es importante hacer notar que el recorte analítico de la ecología de juegos permite reificar a las disputas, ya que las pone en una estructura policéntrica (Lubell, 2013; Smaldino y Lubell, 2014).

En la siguiente sección, se visualizan el conflicto y los diferentes actores en una red de política pública. El análisis realizado utiliza métodos de análisis de discurso y retórica tanto de fuentes primarias y entrevistas con actores clave y expertos que han analizado el caso de El Zapotillo como de fuentes secundarias, en particular reportes de noticias y presentaciones de involucrados en el conflicto en diversos foros.

\section{El conflicto por la presa El Zapotillo como nodo centroidal del conflicto intratable}

Pretender analizar conflictos intratables inevitablemente obliga a la selección de casos de estudio donde el grado de intratabilidad sea visiblemente alto. La disputa por la inundación de tres poblados cercanos al sitio del macroproyecto de la presa El Zapotillo manifiesta claramente un grado alto de intratabilidad, medido de acuerdo con la propuesta descrita en Pacheco-Vega (2014). ${ }^{5}$ Esta sección describe bre-

4. Se debe hacer notar que también hay quien critica la perspectiva de Lubell y colaboradores debido a que el mapeo que se presenta en el recorte analítico de la ecología de juegos no es exclusivamente de actores. Sin embargo, una discusión sobre el tema queda fuera del objetivo del presente trabajo.

5. Ciertamente, medir el grado de intratabilidad es una propuesta un poco arriesgada, pero no imposible. Sin embargo, la medición de manera cuantitativa queda fuera de los objetivos del presente artículo y, por lo tanto, se convierte en objetivo de alguna otra investigación. 
vemente el caso de las confrontaciones y la lucha por parte de activistas, así como las posiciones de los representantes de las agencias gubernamentales relacionadas con el agua, por la construcción de la presa El Zapotillo.

Los orígenes del conflicto se remontan al 23 de febrero de $1990,{ }^{6}$ cuando el entonces gobernador de Guanajuato, Rafael Corrales Ayala, firmó un acuerdo de colaboración con el entonces gobernador de Jalisco, Guillermo Cosío Vidaurri, y con el presidente Carlos Salinas de Gortari, para estudiar las aguas del río Verde. Al conocerse los resultados del estudio, se resolvió, en noviembre de 1994, asignarle 120000000 de $\mathrm{m}^{3}$ a León y 384000000 de $\mathrm{m}^{3}$ a Guadalajara, acuerdo que se oficializó mediante un decreto del Ejecutivo federal publicado en abril de 1995. Este decreto fue modificado el 22 de mayo de 1997, cuando se firmó un nuevo convenio de aprovechamiento integral del río Verde en beneficio de los usuarios de aguas nacionales (Sandoval, 2013).

Luego, el 23 de mayo de 2003, el gobernador de Jalisco, Francisco Ramírez Acuña, publicó un decreto en el Diario Oficial del Estado de Jalisco que autorizó recursos para construir una presa en el río Verde, ubicándose originalmente en el municipio alteño de Jalostotitlán. Se preveía la inundación de las comunidades de San Nicolás y San Gaspar, pero los habitantes de esta última se opusieron tenazmente y en 2004 el gobernador Ramírez Acuña canceló el proyecto (Sandoval, 2013).

Finalmente, en septiembre de 2005 se aprobó la construcción de la presa El Zapotillo en su ubicación actual. También en septiembre, pero de 2007, se firmó el Convenio coordinador para la construcción y operación de la presa, en el que

6. Es de resaltar que el horizonte temporal ha sido sumamente dinámico y determina el grado de intratabilidad dependiendo del momento en el tiempo en el cual se realiza el análisis. Este estudio realiza un análisis desde la propuesta de la presa y del acueducto, pero se ciñe específicamente al conflicto por el acueducto y las discusiones globales una vez que la presa se construyó (julio de 2015). Se agradece al revisor por el comentario. 
se modificó la altura de la cortina de 80 a 105 metros. ${ }^{7}$ En el año 2008, los habitantes de Temacapulín manifestaron por primera vez su rechazo total al proyecto (Cajero, 2008).

A partir de esa fecha, los habitantes inconformes se organizaron en el Comité Salvemos Temacapulín, Acasico y Palmarejo. A través de esta agrupación, han impugnado la licitación (Guillén, 2009), organizado plantones en el sitio de construcción de la presa (Águila, 2008c) y en el predio de Tlalicoyunque, donde se pretende reubicar a los desplazados (El Informador, 2010b), además de organizado manifestaciones en el centro histórico de Guadalajara (Calá, 2009). Han contado con el apoyo de otras organizaciones, como el Comité de Hijos Ausentes de Temacapulín (De Loza, 2011), el Colectivo de Organizaciones Ciudadanas por el Agua (Coloca) (Águila, 2008a) y el Colectivo COA (El Informador, 2009a), por mencionar algunas.

Mientras tanto, los Gobiernos federal y estatal, a través de la Conagua, la Comisión Estatal del Agua (CEA) y el Organismo de la Cuenca Lerma-Chapala-Santiago-Pacífico, han seguido adelante con el proyecto, y en septiembre de 2009 Conagua anunció que La Peninsular Compañía Constructora, en sociedad con FCC Construcción y Grupo Hermes, habían sido los ganadores de la licitación (El Informador, 2009b), teniendo un avance de la construcción de la presa del 54.6\% al 20 de enero de 2013 (De Loza, 2013a). 


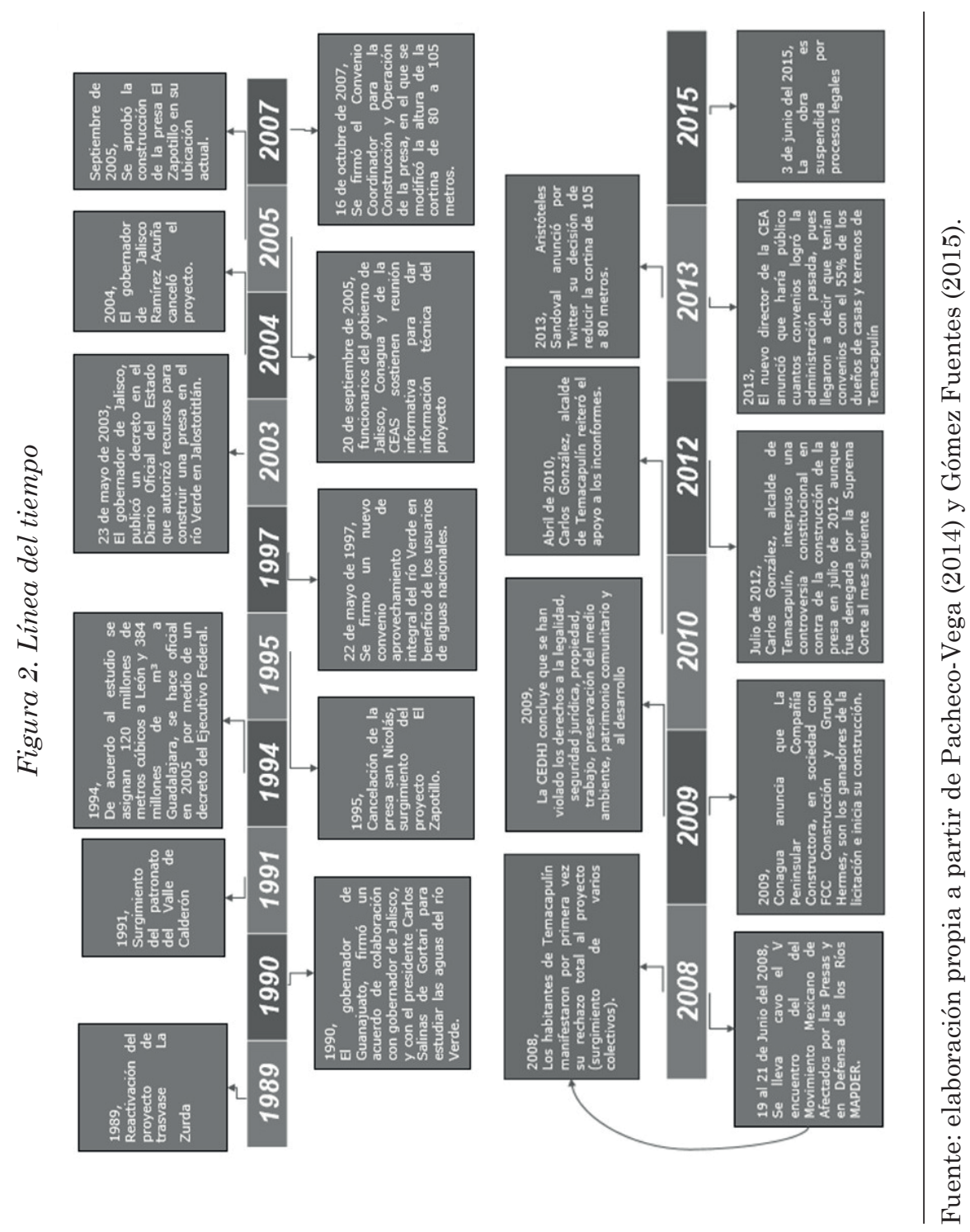

Sociedad $\in$ No.69 
El Gobierno del municipio de Cañadas de Obregón, en cuya zona geografica se ubica el proyecto, argumenta a lo largo del conflicto que nunca ha tenido la información completa acerca de la construcción de la presa. Por esta razón, un alcalde, José de Jesús Sainz, ofreció su apoyo a los habitantes inconformes de Temacapulín, pues estaba en contra de que se inundara este pueblo (Águila, 2008b). Su sucesor en el cargo, Carlos González, reiteró el apoyo a los inconformes en abril de 2010 (El Informador, 2010a) e interpuso una controversia constitucional en contra de la construcción de la presa en julio de 2012 (Guillén, 2012a), misma que fue denegada por la Suprema Corte el mes siguiente (Guillén, 2012b).

Con el cambio de administración estatal, el gobernador Aristóteles Sandoval anunció por Twitter su decisión de reducir la cortina de 105 a 80 metros, tal y como estaba en el proyecto original (De Loza, 2013a). Días después, Sandoval matizó sus comentarios al reconocer que la decisión final de reducir la cortina estaría en manos de la Conagua (El Informador, 2013a).

Para convencer a esta dependencia de la conveniencia del proyecto, el Gobierno de Jalisco está proyectando construir hasta tres presas intermedias, aparte de la presa El Purgatorio, para poder salvar de la inundación a Temacapulín (El Informador, 2013b). Por otra parte, el nuevo director de la CEA anunció que haría público cuanto convenio había logrado la administración pasada de la Comisión (De Loza, 2013b), pues la CEA llegó a decir que tenían convenios con el 55\% de los dueños de casas y terrenos de Temacapulín (El Informador, 2011).

En secciones anteriores, se han postulado tres marcos teóricos para el estudio del conflicto intratable generado por el proyecto de la presa El Zapotillo. Hacer un análisis con cada uno de los mismos resultaría sumamente extenso, por lo que en esta sección únicamente se marcan algunos 
elementos importantes que cada uno de los recortes analíticos aportan (o pueden aportar) al estudio del conflicto.

En primer lugar, desde la perspectiva del marco IER, es claro que la alineación de los marcos perceptuales de los actores en conflicto resulta prácticamente imposible. Las Tablas 1 y 2 usan el modelo presentado en Pacheco-Vega (2014) para analizar conflictos intratables, y de las mismas se puede derivar que el reenmarcamiento de este conflicto resultaría prácticamente imposible.

\section{Tabla 1. Aplicación del modelo IER al conflicto de El Zapotillo}

\begin{tabular}{|c|c|c|c|}
\hline $\begin{array}{l}\text { Caso } \\
\text { Caso de } \\
\text { estudio }\end{array}$ & $\begin{array}{l}\text { Intratabilidad } \\
\text { ¿Es el conflicto } \\
\text { duradero? }\end{array}$ & $\begin{array}{l}\text { Enmarcamiento } \\
\text { ¿Cuáles son los } \\
\text { marcos de los } \\
\text { diferentes actores? }\end{array}$ & $\begin{array}{l}\text { Reenmarcamiento } \\
\text { ¿Cuáles son los } \\
\text { posibles mecanismos } \\
\text { de alineamiento entre } \\
\text { actores? }\end{array}$ \\
\hline $\begin{array}{l}\text { Presa y } \\
\text { acueducto } \\
\text { El Zapotillo }\end{array}$ & Sí & $\begin{array}{l}\text { - Desaparición } \\
\text { por inundación } \\
\text { de los pueblos } \\
\text { de Temacapulín, } \\
\text { Acasico y } \\
\text { Palmarejo, así } \\
\text { como sus tierras } \\
\text { de cultivo. } \\
\text { - Falta de } \\
\text { información hacia } \\
\text { los habitantes } \\
\text { afectados. } \\
\text { - Beneficios para } \\
\text { Guanajuato; } \\
\text { consecuencias } \\
\text { negativas para } \\
\text { Jalisco. }\end{array}$ & $\begin{array}{l}\text { - Puede ser un factor } \\
\text { el mejorar la equidad } \\
\text { por la distribución } \\
\text { de agua al seno de } \\
\text { la cuenca, ya que su } \\
\text { desigual distribución } \\
\text { incrementa la } \\
\text { polarización de los } \\
\text { diferentes actores. } \\
\text { - Sin embargo, los } \\
\text { marcos parecen } \\
\text { extremadamente } \\
\text { polarizados, y por } \\
\text { tanto incompatibles. }\end{array}$ \\
\hline
\end{tabular}

Fuente: elaboración propia a partir de Pacheco-Vega (2014).

En la Tabla 1, se pueden observar los tres momentos del modelo IER en su aplicación al caso de estudio, donde, en 
primer lugar, el conflicto es considerado intratable debido a la duración del mismo; en segundo lugar, los marcos que se observan y que generan que el conflicto se convierta en intratable son: 1) la desaparición de los poblados por inundación, 2) la falta de información hacia los habitantes de las zonas afectadas, y 3) la necesidad de agua para el estado de Guanajuato en oposición a los intereses de los actores afectados en Jalisco; y en tercer lugar, se analizan las posibles maneras de repensar el conflicto y se nota que si bien hay una mejor distribución del agua al seno de la cuenca, se continua percibiendo la polaridad e incompatibilidad de los actores.

En la Tabla 2, se presenta la comparación entre los diferentes grupos de actores involucrados en el conflicto y las afectaciones positivas y negativas, así como las diferencias posicionales de los mismos.

Se consideran como principales actores los municipios afectados por las inundaciones, la Zona Metropolitana de Guadalajara y la Zona Metropolitana de León. La población afectada es principalmente la que vive en cada una de estas regiones (además de la población flotante). Por su parte, los principales actores involucrados dentro del Gobierno son los Gobiernos municipales y estatales, y las instituciones locales y federales como la Conagua, la CEA de Guanajuato y el Organismo de la Cuenca Lerma-Santiago Pacífico. Se puede observar que existe una contraposición no sólo dentro de la sociedad civil, sino también entre los diferentes actores gubernamentales involucrados. 
Tabla 2. Mapeo posicional y de afectaciones por el conflicto de El Zapotillo

Caso:

construcción de

presa y acueducto

El Zapotillo

Población

afectada (número

de habitantes)

Actores

gubernamentales

involucrados en

las negociaciones

\section{Principal \\ elemento de \\ discusión del \\ conflicto}

\section{A \\ Municipios \\ afectados (por \\ inundaciones)}

Negativamente

Temacapulín: 480

Acasico: 365

Palmarejo: 167

además de la

población flotante

Conagua, CEA (de

Guanajuato) y

Organismo de la

Cuenca Lerma-

Santiago Pacífico

Modificación de

la altura de la cortina: pasó de 80 a 105 metros
Posición de los diferentes actores con respecto del conflicto

\section{A favor}

Conagua, CEA y

Organismo de

Cuenca Lerma-

Santiago Pacífico

\section{En contra}

Sociedad civil
$B$

Zona Metropolitana

de Guadalajara

Positivamente

Área metropolitana

de Guadalajara:

950000

Altos de Jalisco:

316000

Gobiernos de los

estados de Jalisco y

Guanajuato

Oposición de los

habitantes de

Temacapulín,

Acasico y Pamarejo a la inundación de sus pueblos y tierras de cultivo

\section{A favor \\ Gobierno del estado de Guanajuato}

\section{En contra}

Gobierno del estado de Jalisco

\section{C}

Zona Metropolitana

de León

Positivamente

Zona Metropolitana

de León: 1095 000*

Gobiernos

municipales de

Cañadas de Obregón, Jalisco, y León, Guanajuato

Guanajuato sería beneficiado, mientras Jalisco pagaría las consecuencias del transvase, de acuerdo con múltiples actores del estado

\section{A favor}

Gobierno del municipio de León

\section{En contra}

Gobierno del municipio de Cañadas de Obregón

* Véase: http://www.guanajuato.gob.mx/ceag/rio-verde.php

Fuente: Pacheco-Vega (2014). ${ }^{8}$

8. Ciertamente es importante hacer notar que los conflictos por la presa y por el acueducto tienen diferentes grados de intensidad y no se encuentran exactamente en el mismo nivel. Como se ha indicado acertadamente, los alteños rechazan el acueducto en este momento, no así la presa, ya que está construida con una altura de 80 metros. Sin embargo, es importante demarcar el grado de conflicto global para no olvidar el posible impacto negativo que ambos megaproyectos 
Cuando se examina el problema mediante el análisis de redes de política pública, se observa que la complejidad del mapeo de la red misma es posiblemente superior a la capacidad analítica que se pudiera invocar con los datos disponibles hasta el momento. Seguramente sería necesario realizar más trabajo de campo y de entrevistas con actores clave, si bien este tipo de esfuerzo pertenece a otro producto de investigación.

En la Figura 3, se realiza una representación visual de los diferentes actores, localizando al conflicto en el nodo centroidal. Se puede observar una categorización de los actores en sociedad civil (trapecios), Gobierno municipal (rectangulos), Gobierno e instituciones estatales (octágonos), Gobierno e instituciones federales (círculos), sector privado (rombos), y Naciones Unidas para Proyectos y Servicios (UNOPS), el área de organizaciones internacionales (nube).

Esta categorización parece relevante puesto que los actores que se encuentran en relación con el conflicto están diferenciados a su naturaleza y tienen diferente peso al interior del mismo.

Por otro lado, se categorizó la postura que toma cada uno de estos actores por medio las flechas, donde las lineas discontinuas representan los actores que se encuentran en contra del proyecto, las líneas solidas representan los actores a favor, y las dobles líneas representan a actores que no tienen una postura definida, o bien, poseen una que ha cambiado de acuerdo con el periodo que se refiera. De ello se puede observar que hay una gran composición de factores de incidencia negativa y pocos de incidencia positiva; otro elemento a reconocer es que, como se observa en la Figura 3, la sociedad civil de León tiene un papel importante en el conflicto (Pacheco-Vega, 2014), sin embargo, su opinión ha sido invisibilizada. 


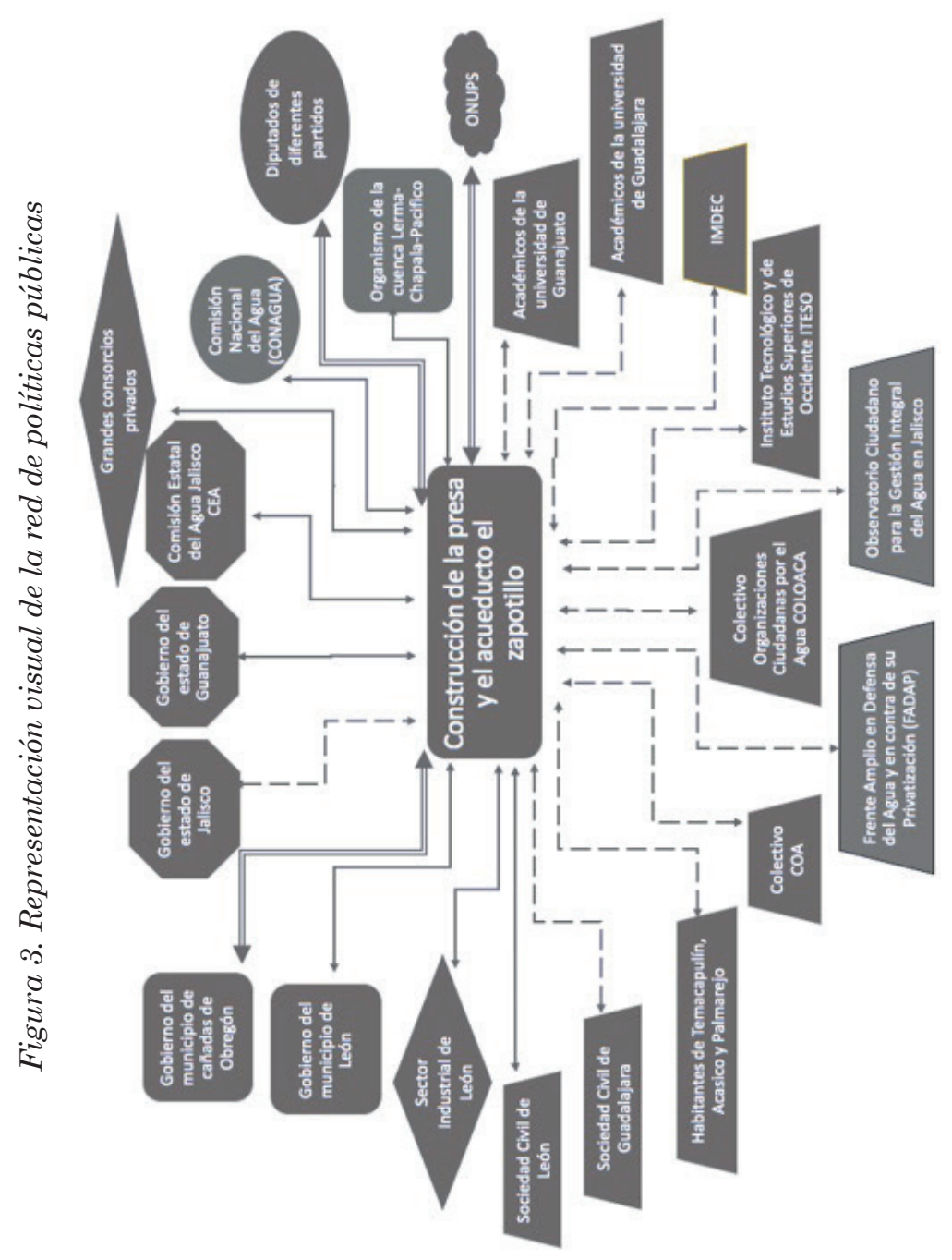

Sociedad $\in$ No.69 
Los habitantes de Temacapulín, pronunciados en diferentes maneras y momentos por la preservación de los pueblos amenazados, consideran que la industria de la construcción y del calzado de León quiere el agua para continuar con sus actividades, aunque esto represente la contaminación por cromo y bromo, elementos muy utilizados en sus procesos.

La justificación de la construcción de la presa El Zapotillo es abastecer de agua a la ciudad de León, Guanajuato, a la Zona Metropolitana de Guadalajara y a la región de Los Altos de Jalisco (Vera, 2015). Sin embargo, compañías nacionales e internacionales de la agroindustria y la industria del calzado serán quienes reciban el mayor abastecimiento de agua proveniente del estado de Jalisco, respaldadas por el Gobierno de Guanajuato desde el convenio firmado en 2009. Por su parte, empresas instaladas dentro del llamado "puerto seco" buscan distribuir, producir y comercializar sus productos como lo han venido realizando, y para garantizarlo necesitan el agua de Jalisco (Vera, 2015).

Si se reflexiona sobre la justificación, claramente se tendría el resultado de que dicho argumento es falso, pues no toda la ciudad se dedica a la industria y no toda la industria abarca la sociedad civil de León. Aunque los ciudadanos de dicha ciudad se pronunciaran a favor, estarían actuando bajo lo que se dice y se sabe de los acuerdos y convenios, sin embargo, se verían favorecidos en un periodo de tiempo indefinido. Es decir, en caso de que estuvieran de acuerdo con lo pactado, sólo estarían actuando en favor de terceros, quienes, a su vez, están ampliamente respaldados por el Gobierno.

Esto es interesante, pero no sorprendente, dadas las percepciones con respecto de lo que realmente se obtiene en la ciudad de León del megaproyecto: un incremento en el suministro de agua potable disponible para utilización urbana e industrial, a pesar de la nula eficiencia en utilización del recurso hídrico en dicha urbe (Tagle Zamora, 2014).

\section{8}


Durante los últimos meses, un par de actores que se han vuelto claves en la trayectoria que ha tomado el conflicto es UNOPS y el Programa de las Naciones Unidas para el Medio Ambiente, quienes en principio deberían operar como mediadores del conflicto entre la sociedad civil que se opone al proyecto y los diferentes órdenes e instituciones de Gobierno que han sido los encargados de la toma de decisiones. En ese sentido, podrían ser un elemento que reacomodara las relaciones de desequilibrio entre los pueblos afectados por las obras y los órdenes de Gobierno e instituciones.

A este respecto, UNOPS está realizando un estudio a partir de una solicitud que realizó el estado de Jalisco, con una duración de dieciocho meses. Los fondos para la realización del estudio son otorgados por el estado y el proyecto tiene la intención de hacer un diagnóstico neutral del conflicto que permita definir la viabilidad del proyecto. ${ }^{9}$ Sin embargo, se observa un descontento por parte de los habitantes de estas zonas hacia el trabajo que esta institución se encuentra realizando en el lugar, ya que de manera fundada o infundada se han cuestionado los objetivos de la institución en el estudio que realiza. Así, diferentes notas de periódico muestran cómo se coloca en duda si es una institución que únicamente tiene la intención económica de realizar el estudio y si el Gobierno de Jalisco está solicitando este estudio únicamente como manera de validar el objetivo que siempre ha mantenido, que es la construcción de la presa en las condiciones que se plantearon originalmente. ${ }^{10}$

9. Véase: https://www.unops.org

I0. Véase: http://www.ntrguadalajara.com/post.php?id_nota $=52944$ 
Conclusiones: el conflicto intratable por la presa

El Zapotillo visto desde la ecología política urbana

El conflicto intratable por el proyecto del acueducto y presa de El Zapotillo que aquí se analiza ofrece una gran cantidad de datos empíricos, particularmente porque facilita el mapeo de las diferentes posiciones en conflicto (PachecoVega, 2014), así como el esclarecimiento de los elementos que llevan a la divergencia de posiciones ideológicas. ${ }^{11}$

En este último apartado, se concluye al resaltar la contribución de la ecología política como lente analítico para estudiar conflictos intratables. Al poner de relieve el impacto negativo que tiene la imposición de estrategias capitalistas, comercializadoras y mercantilizadoras en el manejo del agua, la ecología política permite explicar de manera empírica la disparidad de poderes que tienen los diferentes nodos de la red de política pública.

Asimismo, la ecología política pone de relieve las diferentes razones por las cuales un conflicto se puede volver intratable dadas las disparidades de poder e impacto en el diseño de las políticas. La ecología política permite visibilizar estas disparidades y ponerlas bajo gran variedad de diferentes lentes analíticos que pueden ser de utilidad para el diseño e implantación de políticas hídricas.

En este artículo se ha tomado una visión desde la ecología política para analizar el conflicto intratable de la presa El Zapotillo, tratando de examinar las relaciones de poder y las desigualdades que se acentúan en un contexto en el cual las respuestas tecnocráticas para resolver problemas de estrés hídrico provienen de una serie de instituciones jerárquicas y de una estructura sistémica que toma decisiones de gobernanza del recurso hídrico desde la cúpula y

II. Porciones de dicho ejercicio analítico fueron tomadas de otro documento ya publicado. 
no desde la base, tomando en cuenta las opiniones de las comunidades a las cuales afectan dichas decisiones.

Como demuestra el análisis presentado, la expansión de la huella ecológica de la Zona Metropolitana de León, específicamente de la ciudad, ha no solamente transformado el territorio en la región vecina a esta urbe del estado de Guanajuato, sino que también ha incrementado de manera artificial la demanda del recurso, lo que ha dado lugar a un conflicto cuya naturaleza se ha convertido en intratable. Este caso es similar a lo que ha ocurrido en España (Swyngedouw, et al., 2002; Swyngedouw y Heynen, 2003) con la creación de redes interregionales de manejo de agua que alimentan un sistema nacional.

En el artículo se aplicó el modelo IER para explicar cómo la disputa por el agua entre Jalisco y Guanajuato se ha convertido en intratable. Los conflictos intratables surgen cuando una confrontación tiene un tiempo de resolución muy largo, continúa en proceso de negociación, no parece tener solución negociada de manera visible y sencilla, y presenta posiciones contrapunteadas entre los actores en discordia que no muestran resolución en el corto o mediano plazo, con desacuerdos constantes. Como se ha demostrado en el artículo, las disputas por la construcción del megaproyecto El Zapotillo y la inundación de tres poblados en Jalisco han resultado ser intratables. Al analizar los diferentes marcos y perspectivas que cada actor o grupo de actores involucrados en la disputa tienen, se encuentra que es prácticamente imposible diseñar un modelo cooperativo en el cual los marcos se podrían alinear para ofrecer una solución negociada al conflicto. $\_$ 
Bibliografía
Águila, E. (II de septiembre de 2008a). "Rechazan organizaciones presas de Arcediano y El Zapotillo”. El Informador. Recuperado de:http://www.informador.com.mx/ jalisco/2008/37707/6/rechazan-organizaciones-presasde-arcediano-y-el-zapotillo.htm

(0I de octubre de 2008b). "Alcalde de Cañadas de Obregón desconoce proyecto El Zapotillo”. El Informador. Recuperado de: www.informador.com.mx/ jalisco/2008/42662/I/alcalde-de-canadas-de-obregondesconoce-proyecto-el-zapotillo.htm

(II de diciembre de 2008c). "Impiden pobladores recorrido de empresas por presa El Zapotillo”. El Informador. Recuperado de: www.informador.com.mx/ jalisco/2008/6 I 720/ //impiden-pobladores-recorridode-empresas-por-presa-el-zapotillo.htm

Aguilera-Klink, F., Pérez-Moriana, E., y Sánchez-García, J. (2000)."The social construction of scarcity. The case of water in Tenerife (Canary Islands)". Ecological Economics, 34(2), 233-245. doi: I0.10I6/S092I-8009(00)00160-9

Alonso, R. (08 de abril de 2015). "Bajo la lupa: presa El Zapotillo, proyecto hídrico en debate”. Bnamericas. Recuperado de: http://www.bnamericas.com/es/news/ aguasyresiduos/bajo-la-lupa-presa-el-zapotillo-unproyecto-hidrico-en-disputa I

Bakker, K. J. (2003). "A Political Ecology of Water Privatization”. Studies in Political Economy, (70), 35-58. Recuperado de: http://spe.library.utoronto.ca/index.php/spe/issue/ view/912

Bar-Tal, D. (2000). "From Intractable Conflict Through Conflict Resolution To Reconciliation: Psychological Analysis". Political Psychology, 2 I (2), 35I-365.

Becerra Pérez, M., Sáinz Santamaría, J., y Muñoz Piña, C. (2006).“Los conflictos por agua en México. Diagnóstico y análisis”. Gestión y Política Pública, XV(I), I I I-I43. 
Berardo, R. (2009). "Processing Complexity in Networks:A Bibliografía Study of Informal Collaboration and its Effect on Organizational Success". Policy Studies Journal, 37(3), 52 I-539. doi: I 0. I I I I/j. I 54 I-0072.2009.00326.x

Beyers, J., y Braun, C. (20I4). "Ties that count: explaining interest group access to policymakers". Journal of Public Policy, 34(0I), 93-I2I.doi:I0.10I7/ S0I438I4XI3000263

Biro, A. (2007). "Water politics and the construction of scale". Studies in Political Economy, 80, 9-30. Recuperado de: http://spe.library.utoronto.ca/index.php/spe/article/ view/5 I I3/I965

Burgess, H., y Burgess, G. M. (2003). "What Are Intractable Conflicts?”, en G. Burgess, y H. Burgess (eds.), Beyond Intractability, Boulder Conflict Information Consortium. Boulder:University of Colorado. Recuperado de: http://www. beyondintractability.org/bi-essay/meaning-intractability

Cairé Martínez, G. L. (2005). "Conflictos por el agua en la cuenca Lerma-Chapala, 1996-2002”. Región y Sociedad, XVII(34), 73-I25. Recuperado de: http://www.redalyc. org/articulo.oa?id= 10203403

Cajero, L. (20 de noviembre de 2008). "Pese a reubicación digna, se manifiestan contra El Zapotillo". El Informador. Recuperado de www.informador.com.mx/ jalisco/2008/55976/I/pese-a-reubicacion-digna-semanifiestan-contra-el-zapotillo.htm

Calá, C. (2I de marzo de 2009). “Protestan frente a Palacio de Gobierno". El Informador. Recuperado de: www. informador.com.mx/jalisco/2009/88/34/I/protestanfrente-a-palacio-de-gobierno.htm

Cohen, A., y McCarthy, J. (20I4). "Reviewing rescaling: Strengthening the case for environmental considerations”. Progress in Human Geography, 39(I), 3-25. doi:I0.II77/0309|325|452|483

De Loza, I. (06 de junio de 20II). "Lucha contra EI Zapotillo recibe apoyo internacional”. El Informador. 
Bibliografía
Recuperado de: http://www.informador.com.mx/ jalisco/20 I I/297900/ I/lucha-contra-el-zapotillo-recibeapoyo-internacional.htm

(29 de enero de 20 I 3a).“Temacapulín no se inunda: Aristóteles”. El Informador. Recuperado de: http://www. informador.com.mx/ jalisco/20/3/433573/6/temacapulin-no-se-inunda-aristoteles.htm

(22 de marzo de 20I3b). “'Nueva’ CEA ventilará convenios entre el Estado y Temacapulín”. El Informador. Recuperado de: www.informador.com.mx/ jalisco/20 I3/446203/I/nueva-cea-ventilara-conveniosentre-el-estado-y-temacapulin.htm

Debbané, A.M.(20/3).“Dis/articulations and the hydrosocial cycle: postapartheid geographies of agrarian change in the Ceres Valley, South Africa". Environment and Planning A, 45(I I), 2553-257I. doi: I0.1068/a45693

Domene Gómez, E. (2006). "La ecología política urbana: una disciplina emergente para el análisis del cambio socioambiental en entornos ciudadanos". Documents $d$ Analisi Geograftca, 48(I), 167-I78.

El Informador (30 de enero de 2009a). "Afectados por presa El Zapotillo pretenden defensa internacional". Recuperado de: http://www.informador.com.mx/ jalisco/2009/744 | 4/ I/afectados-por-presa-el-zapotillopretenden-defensa-internacional.htm

- (I5 de septiembre de 2009b). "Ya hay constructor para El Zapotillo". Recuperado de: www.informador. com.mx/jalisco/2009// 37657/I/ya-hay-constructor-para-el-zapotillo.htm

(27 de abril de 2010a). "Alcalde de Cañadas de Obregón reitera apoyo a Temaca”. Recuperado de:www. informador.com.mx/jalisco/20 I 0// 9684I/// alcalde-decanadas-de-obregon-reitera-apoyo-a-temaca.htm (09 de noviembre de 20l0b). "Pobladores de Temacapulín impiden acceso a nuevo centro de 
población". Recuperado de: www.informador.com.mx/ Bibliografía jalisco/20 10/247530/1/pobladores-de-temacapulinimpiden-acceso-a-nuevo-centro-de-poblacion.htm ( 15 de septiembre de 20 I I).“La CEA anuncia $55 \%$ de convenios con pobladores de Temacapulín”. Recuperado de: www.informador.com.mx/jalisco/20 I l/322390/l/ la-cea-anuncia-55-de-convenios-con-pobladores-detemacapulin.htm

(08 de febrero de 2013a). "El Zapotillo, en manos de Conagua”. Recuperado de: http://www.informador. com.mx/ jalisco/2013/435776/6/el-zapotillo-en-manosde-conagua.htm

(26 de marzo de 20l3b). "El Purgatorio puede dar entre dos y tres mil litros de agua a la ciudad". Recuperado de:www.informador.com.mx/jalisco/20 I3/44694I/l/elpurgatorio-puede-dar-entre-dos-y-tres-mil-litros-deagua-a-la-ciudad.htm

Elgin, D. J. (20I5). "Utilizing Hyperlink Network Analysis to Examine Climate Change Supporters and Opponents". Review of Policy Research, 32(2), 226-245.

Elliott, M., Gray, B., y Lewicki, R. J. (2003). “Lessons Learned about the Framing and Reframing of Intractable Environmental Conflicts", en R. J. Lewicki, B. Gray, y M. Elliott (eds.), Making Sense of Intractable Environmental Conflicts. Concepts and Cases (pp. 409-436). Washington: Island Press.

Emery, S. B., Perks, M.T., y Bracken, L.J. (20I3).“Negotiating river restoration: The role of divergent reframing in environmental decision-making”. Geoforum, 47(I), I67177.

Furlong, K. (2012). “Good water governance without good urban governance? Regulation, service delivery models, and local government". Environment and Planning A, 44 (I I), 272I-274I. doi: I0.1068/a446I6 
Bibliografía
Gandy, M. (2006). “The Bacteriological City and Its Discontents”. Historical Geography, 34, 14-25.

Gómez Fuentes, A. (20I5). "Análisis cronológico por la construcción de la presa el Zapotillo (2005-20I4)". Waterlat-Gobacit Network, 2(I), 246-268.

Gray, B. (2003). "Framing of Environmental Disputes", en R. J. Lewicki, B. Gray, y M. Elliott (eds.), Making Sense of Intractable Environmental Conflicts. Concepts and Cases (pp. I I-34).Washington: Island Press.

Guillén, A. (I 3 de febrero de 2009). “Impugnan licitación para El Zapotillo”. El Informador. Recuperado de: www. informador.com.mx/jalisco/2009/7856 // //impugnanlicitacion-para-el-zapotillo.htm

- (24 de julio de 2012a). "Cañadas interpone controversia constitucional por El Zapotillo”. El Informador. Recuperado de: http://www.informador.com.mx/ jalisco/2012/392 I89/6/canadas- interpone-controversiaconstitucional-por-el-zapotillo.htm

(20 de agosto de 20I2b). "La SCJN niega suspensión de presa El Zapotillo". El Informador. Recuperado de: www.informador.com.mx/jalisco/20 I 2/398933/ //lascjn-niega-suspension-de-presa-el-zapotillo.htm

Heikkila, T., y Schlager, E. C. (20I2). “Addressing the Issues: The Choice of Environmental Conflict-Resolution Venues in the United States". American Journal of Political Science, 56(4), 774-786.

Lewicki, R.J., Gray, B.,y Elliott, M. (eds.) (2003). Making Sense of Intractable Environmental Conflicts: Concepts and Cases. Washington: Island Press.

Linton,J. (20I4)."Modern water and its discontents: a history of hydrosocial renewal". Wiley Interdisciplinary Reviews: Water, I ( I), I I I-I 20. doi: 10.1002/wat2. 1009

Loftus, A. J., y McDonald, D. A. (200I). "Of liquid dreams: a political ecology of water privatization in Buenos 
Aires". Environment and Urbanization, 13(2), 179-199. doi:I0.1 I77/0956247801013002I5

Lubell, M. (2013). "Governing institutional complexity: The ecology of games framework”. Policy Studies Journal, 4 I (3), 537-559. doi: I0.1 I I I/psj. 12028

- (2015). "Collaborative partnerships in complex institutional systems". Current Opinion in Environmental Sustainability, I 2, 4I-47. doi: I0.10 I6/j.cosust.2014.08.0 I I Mahon, R.,y Keil, R. (2009).“Introduction”, en R. Mahon, y R. Keil (eds.), Leviathan Undone? Towards a Political Economy of Scale (pp. 3-26).Vancouver:The University of British Columbia Press. doi: I0.1503/cmaj. I0II75

Mamudu, H., Cairney, P., y Studlar, D. (20I5). “Global Public Policy: Does the New Venue for Transnational Tobacco Control Challenge the OldWay of Doing Things?". Public Administration, 93(4), 856-873. doi: I 0. I I I I/padm. I 2 I 43 Marsh, D. (ed.) (1998). Comparing Policy Networks. Buckingham-Philadelphia: Open University Press.

Monstadt, J. (2009). "Conceptualizing the political ecology of urban infrastructures: Insights from technology and urban studies". Environment and Planning A, 4I, 19241942. doi: |0.1068/a4|45

Oswald Spring, U. (2006)."Hidrodiplomacia y conflictos por el acceso al agua", en V. Vázquez Garcia, y D. Soares (eds.), Gestión y Cultura del Agua.Tomo 2 (pp. 22-6I). Jiutepec, Morelos: Instituto Mexicano de Tecnología del Agua.

Pacheco-Vega, R. (20I4). “Conflictos intratables por el agua en México: aplicando el recorte analítico de intratabilidad, enmarcamiento y reenmarcamiento (IER)", en F. de Alba, y L. Amaya (eds.), Estado y ciudadanía del agua:cómo significar las nuevas relaciones (PP. 279-3 I7). México:UAM. Park, H. H., y Rethemeyer, R. K. (20I4). "The politics of connections: Assessing the determinants of social structure in policy networks". Journal of Public Adminis- 
Bibliografía tration Research and Theory, 24, 349-379. doi:I0.1093/ jopart/ mus02I

Paz Salinas, M. F. (2012). "Deterioro y resistencias: conflictos socioambientales en México", en D. Tetreault, $\mathrm{H}$. Ochoa Garcia, y E. Hernández González (eds.), Conflictos Socioambientales y Alternativas de la Sociedad Civil (pp. 27-48). Guadalajara: ITESO.

Pralle, S. B. (2003). "Venue Shopping, Political Strategy, and Policy Change: The Internationalization of Canadian Forest Advocacy”. Journal of Public Policy, 23(3), 233-260. Putnam, L. L., y Wondolleck, J. M. (2003).“'Intractability: Definitions, Dimensions, and Distinctions", en R. J. Lewicki, B. Gray, y M. Elliott (eds.), Making Sense of Intractable Environmental Conflicts. Concepts and Cases (Pp. 35-62). Washington: Island Press.

Sandoval, A. (I 8 de febrero de 20 I3).“"El Zapotillo, 23 años en busca de agua para León”. Periódico Correo. Recuperado de: www.periodicocorreo.com.mx/especiales/84566el-zapotillo-23-anos-en-busca-de-agua-para-leon.html

Schafer, J. G. (20I2). A Flower Blooms in the Desert:Managing for Collaboration. Las Vegas: University of Nevada.

Smaldino, P. E., y Lubell, M. (20/4). "Institutions and Cooperation in an Ecology of Games". Artificial Life, 20(2), 207-22 I.doi:I0.I I62/ARTL_a_00I 26

Swyngedouw, E., y Heynen, N. C. (2003). “Urban Political Ecology, Justice and the Politics of Scale". Antipode, 35 (5), 898-9 |8. doi: I 0. I I I I/j. I 467-8330.2003.00364.x

Swyngedouw, E., Kaïka, M., y Castro, E. (2002).“Urban Water: A Political-Ecology Perspective”. Built Environment, 28 (2), I24-I37.

Tagle Zamora, D. (ed.) (20I4). La crisis multidimensional del agua en la ciudad de León, Guanajuato. Guanajuato: Universidad de Guanajuato.

Vargas-Velázquez, S., Mollard, E., y Guitrón de los Reyes, A. (eds.) (20I2). Los conflictos por el agua en México: 
El megaproyecto de la presa El Zapotillo como nodo centroidal de conflicto intratable. Un análisis desde la ecología política

caracterización y prospectiva. Jiutepec, Morelos: Instituto Bibliografía Mexicano de Tecnología del Agua.

Vera, L. D. (20I5).“Presa El Zapotillo;Jalisco, México. La resistencia de Temacapulín ante un proyecto extractivista inmerso en irregularidades e impunidad del Gobierno mexicano". EntreTextos, 6(18), 10.

Weible, C. M., y Sabatier, P. A. (2005). "Comparing policy networks: Marine protected areas in California". Policy Studies Journal, 33(2), I8I-20I. doi:I0.I I I //j.I54I$0072.2005 .00101 . x$ 